\title{
STRAIN ENERGY DENSITY RATIO CRITERION FOR FRACTURE OF COMPOSITE MATERIALS
}

\author{
S. Y. ZHANG, $\dagger \ddagger$ L. W. TSAI† and J. Q. LIUß \\ ¥Institute of Mechanics, Chinese Academy of Sciences, Beijing, 100080, People's Republic of \\ China. §Beijing Institute of Technology, Beijing, People's Republic of China

\begin{abstract}
The strain energy density ratio (SEDR) criterion for fracture of fibre reinforced composite materials is proposed. The definition and expression of the SEDR are presented first, then its function of predicting crack growth direction in the materials is demonstrated through the comparison of the experimental and predicted results. In the tests, the crack directions, both in initiation stage and in the pre-fracture propagation stage, were recorded with Moire's fringe method and the ordinary photography. Finally, the function of SEDR criterion for predicting failure strength of fibre composites was illustrated through the comparison of the predicted results of SEDR and Tsai-Hill criteria.
\end{abstract}

\section{INTRODUCTION}

IN AN ISOTROPIC material, the crack of mode-I always propagates self-similarly. However, a mixed-mode crack will propagate in the direction at an angle to its original direction. Thus, besides fracture stress, the prediction of cracking direction is an important subject of LEFM. So far quite a few criteria have been established[1], among them, the maximum tangential stress criterion and the strain energy density (SED) criterion are the two of the most commonly used.

For the continuous fibre reinforced composites, only in rare cases, say mode-I parallel to the fibres, can the crack grow self-similarly, whereas in most cases, the crack will extend in a mixed-mode fashion. Thus, to know the cracking direction is significant to understand the fracture mechanism and for the reliable design of the structure composed of this kind of material. For this purpose, Gregory and Herakovich[2] proposed the normal stress ratio criterion (NSR) to predict crack growth direction. The SED criterion has also been used to analyse crack problems in composite materials[2,3].

Fibre reinforced composites possess strong anisotropy in its fracture toughness as well as in stiffness and strength properties. This characteristic is clearly typified by the unidirectional fibre composites. The fracture toughness of the crack parallel to the fibres, $K_{f c}^{L}$ is much smaller than that of the crack normal to the fibres, $K_{k c}^{T}$, although for the latter configuration, cracking direction is still along fibres. Experimental evidence indicates that for commonly used composites, $K_{i c}^{T} / K_{L c}^{L}=3 \sim 10[4]$. As the SED criterion takes no account of the fracture and strength anisotropy of the materials, it cannot yield good prediction.

In the previous paper of the present authors[5], an attempt was made to predict the cracking direction in the lap-shear specimen of chopped strand mat glass fibre reinforced polyester undergoing the interlaminar shear fracture test. The results predicted by the SEDR criterion proved to be quite successful. To further ascertain the validity of the SEDR criterion, in the present paper, it is applied to predict the crack growth direction in the edge notched sheet specimens of different kinds of composite materials, which exhibit a wide range of anisotropy. Fracture experiments were conducted and the crack initiation and propagation directions were recorded with Moire method and the ordinary photography. The observed results were in good agreement with the predicted ones.

In addition, the SEDR criterion can also be used as failure criterion of predicting the failure strength of plain specimens without notch, crack or other discontinuities. The final part of the paper will describe this function with the example of off-axial tension strength of unidirectional composites. 


\section{THE STRAIN ENERGY DENSITY RATIO CRITERION}

\subsection{Definition and expression}

The strain energy density ratio (SEDR) is defined as the strain energy density calculated from stress ratios and strain ratios. The stress ratios are the ratios of stress values to their respective strength values:

$$
\begin{aligned}
& \bar{\sigma}_{1}=\sigma_{1} / X \\
& \bar{\sigma}_{2}=\sigma_{2} / Y \\
& \bar{\tau}_{12}=\tau_{12} / S
\end{aligned}
$$

where $X$ and $Y$ are the tensile strengths in 1 and 2 directions respectively and $S$ is shear strength. The strain ratios are defined as the ratios of strain values to their respective critical values:

$$
\begin{gathered}
\bar{\varepsilon}_{1}=\varepsilon_{1} / \varepsilon_{1 c} \\
\bar{\varepsilon}_{2}=\varepsilon_{2} / \varepsilon_{2 c} \\
\tilde{\gamma}_{12}=\gamma_{12} / \gamma_{12 c}
\end{gathered}
$$

where $\varepsilon_{1}$ and $\varepsilon_{2}$ are normal strains in 1 and 2 directions respectively; $\gamma_{12}$ is shear strain; and $\varepsilon_{1 c}$, $\varepsilon_{2 c}$ and $\gamma_{12 c}$ are their critical values. For the standard tensile specimen, the critical strain values can be obtained from the strengths as follows:

$$
\begin{aligned}
\varepsilon_{1 c} & =X / E_{11} \\
\varepsilon_{2 c} & =Y / E_{22} \\
\gamma_{12 c} & =S / G_{12} .
\end{aligned}
$$

Here, the assumption of linear elastic relationship between stress and strain components is used. Thus, the expression of SEDR can be calculated as:

$$
S_{R}=\frac{1}{2}\left(\bar{\sigma}_{1} \bar{\varepsilon}_{1}+\bar{\sigma}_{2} \bar{\varepsilon}_{2}+\bar{\tau}_{12} \bar{\gamma}_{12}\right)
$$

Substituting eqs (1) and (2) into eq. (4) and referring to eq. (3), we obtained:

$$
S_{R}=\frac{1}{2}\left[\left(\frac{\sigma_{1}}{X}\right)^{2}+\left(\frac{\sigma_{2}}{Y}\right)^{2}-\frac{v_{12}}{E_{11}} \sigma_{1} \sigma_{2}\left(\frac{E_{11}}{X^{2}}+\frac{E_{22}}{Y^{2}}\right)+\left(\frac{\tau_{12}}{S}\right)^{2}\right] .
$$

\subsection{The SEDR criterion for cracking direction}

The SEDR criterion stipulates that the cracking will occur in the radial direction along which the local SEDR value reaches its minimum value, i.e.

$$
\frac{\partial S_{R}}{\partial \theta}=0 \text { and } \quad \frac{\partial^{2} S_{R}}{\partial \theta^{2}}>0 \quad \text { when } \theta=\theta_{\mathrm{c}}
$$

If there are more than one minimum values around the point concerned, the cracking direction will coincide with the maximum one, thus,

$$
S_{R}=\left(S_{R}\right)_{\min }^{\max } \quad \text { when } \theta=\theta_{c} .
$$

\subsection{The $S E D R$ criterion for failure strength}

This criterion assumes that when double of the SEDR value is equal to or larger than unity the material will fail. Thus, the failure condition can be expressed as:

$$
2 S_{R} \geqslant 1, \quad \text { or }\left(\frac{\sigma_{1}}{X}\right)^{2}+\left(\frac{\sigma_{2}}{Y}\right)^{2}-\frac{\nu_{12}}{E_{11}} \sigma_{1} \sigma_{2}\left(\frac{E_{11}}{X^{2}}+\frac{E_{22}}{Y^{2}}\right)+\left(\frac{\tau_{12}}{S}\right)^{2} \geqslant 1
$$




\section{NUMERICAL ANALYSIS AND EXPERIMENTAL VERIFICATION}

\subsection{Computational procedure}

The computational model is as shown in Fig. 1. $\beta$ is the first principal direction of the material, that is the direction of fibres for an unidirectional composite. $\theta$ is the argument of the polar coordinate. From anisotropic linear elastic fracture mechanics, we have the following three formulas of the three stress components in the near crack zone.

$$
\begin{gathered}
\sigma_{x}=\frac{\sigma^{\infty} \sqrt{a}}{\sqrt{2 r}} \operatorname{Re}\left\{\frac{\mu_{1} \mu_{2}}{\mu_{1}-\mu_{2}}\left[\frac{\mu_{2}}{\psi_{2}}-\frac{\mu_{1}}{\psi_{1}}\right]\right\} \\
\sigma_{y}=\frac{\sigma^{\infty} \sqrt{2}}{\sqrt{2 r}} \operatorname{Re}\left\{\frac{1}{\mu_{1}-\mu_{2}}\left[\frac{\mu_{1}}{\psi_{2}}-\frac{\mu_{2}}{\psi_{1}}\right]\right\} \\
\tau_{x y}=\frac{\sigma^{\infty} \sqrt{a}}{\sqrt{2 r}} \operatorname{Re}\left\{\frac{\mu_{1} \mu_{2}}{\mu_{1}-\mu_{2}}\left[\frac{1}{\psi_{1}}-\frac{1}{\psi_{2}}\right]\right\}
\end{gathered}
$$

where

$$
\begin{aligned}
& \psi_{1}=\left(\cos \theta+\mu_{1} \sin \theta\right)^{1 / 2} \\
& \psi_{2}=\left(\cos \theta+\mu_{2} \sin \theta\right)^{1 / 2} .
\end{aligned}
$$

In the above equations, $\mu_{1}$ and $\mu_{2}$ (and their conjugates $\bar{\mu}_{1}$ and $\bar{\mu}_{2}$ ) are two unequal roots of the following characteristic equation:

$$
A_{11} \mu^{4}-2 A_{16} \mu^{3}+\left(2 A_{12}+A_{66}\right) \mu^{2}-2 A_{26} \mu+A_{22}=0
$$

where $A_{i j}$ are the coefficients of compliance matrix. Substituting the three stress components in eq. (9) into eq. (5) and assigning for simplicity $\sigma^{\infty}=0.1 \mathrm{MPa}, r=0.1 \mathrm{~mm}, a=20 \mathrm{~mm}$, straightforward calculation yields $S_{R}-\theta$ curves, from which the value of $\left(S_{R}\right)_{\min }^{\max }$ and the corresponding critical angle $\theta_{c}$ can be determined. At first, we calculated the $S_{R}-\theta$ curves for three cases of the fibre angles being equal to $5^{\circ}, 45^{\circ}$ and $105^{\circ}$. The results are as shown in Fig. 2. The material constants are: $E_{11}=151.9 \mathrm{GPa}, E_{22}=13.7 \mathrm{GPa}, v_{12}=0.28, G_{12}=5.83 \mathrm{GPa}, X=1.983 \mathrm{GPa}, \quad Y=0.0703 \mathrm{GPa}$,

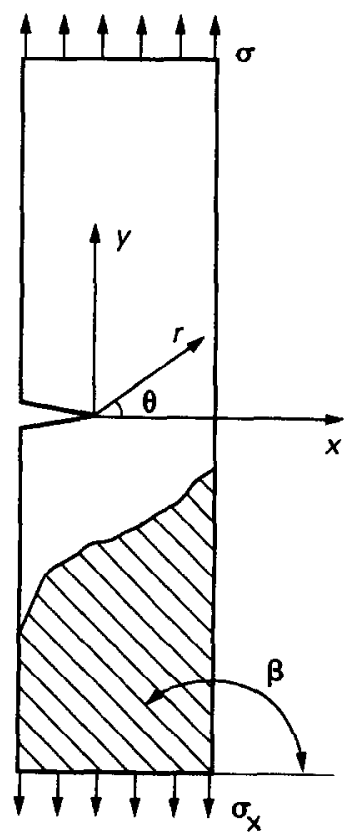

Fig. 1. Computation model.

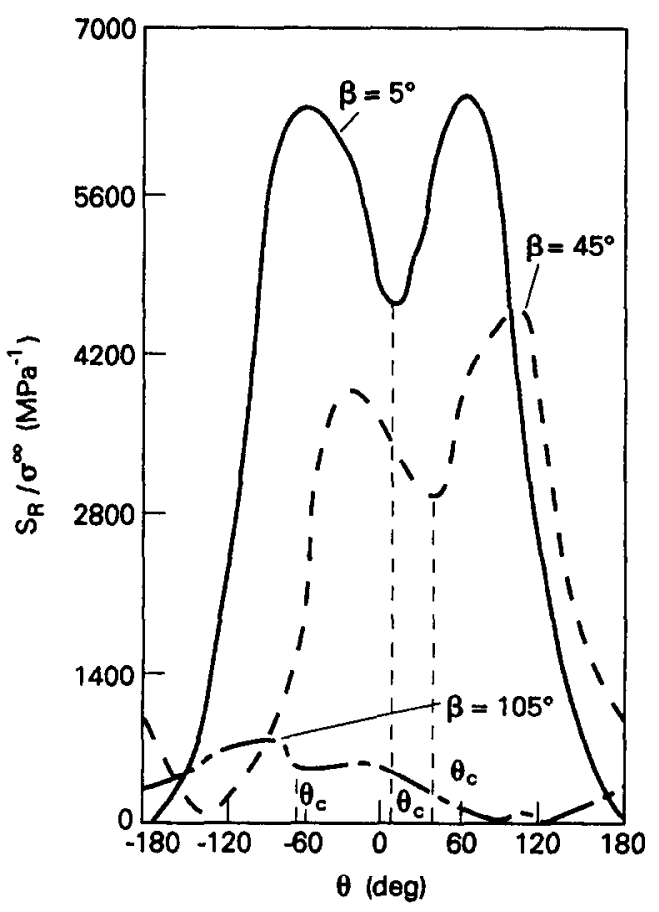

Fig. 2. Variation of $S_{R}$ as a function of $\theta$, for $\beta=5^{\circ}, 45^{\circ}$ and $105^{\circ}$. 


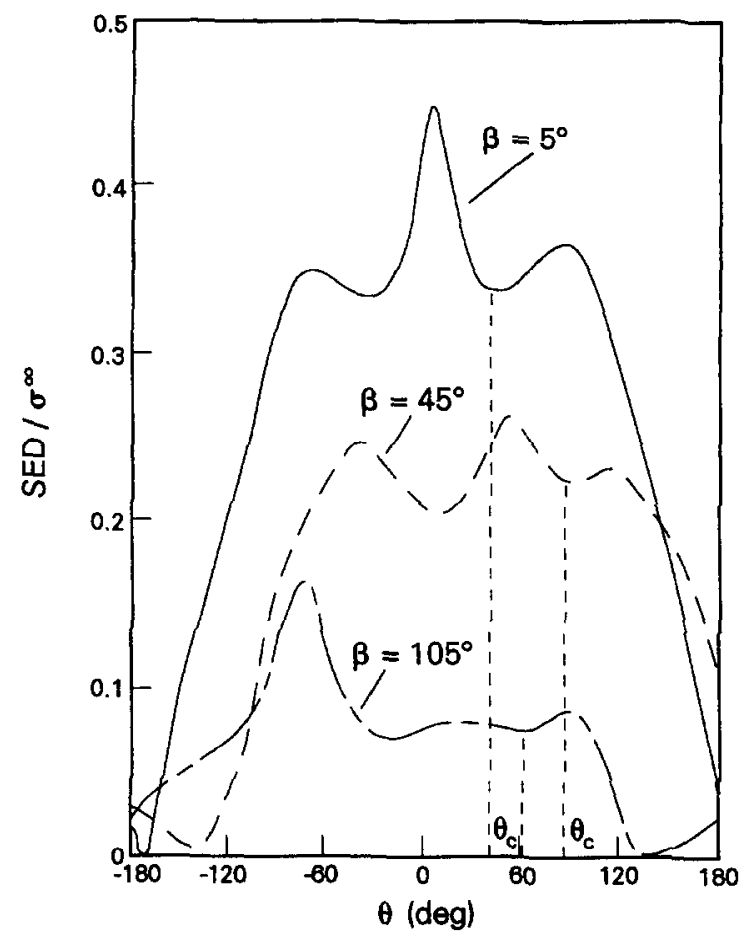

Fig. 3. Variation of SED as a function of $\theta$, for $\beta=5^{\circ}, 45^{\circ}$ and $105^{\circ}$.

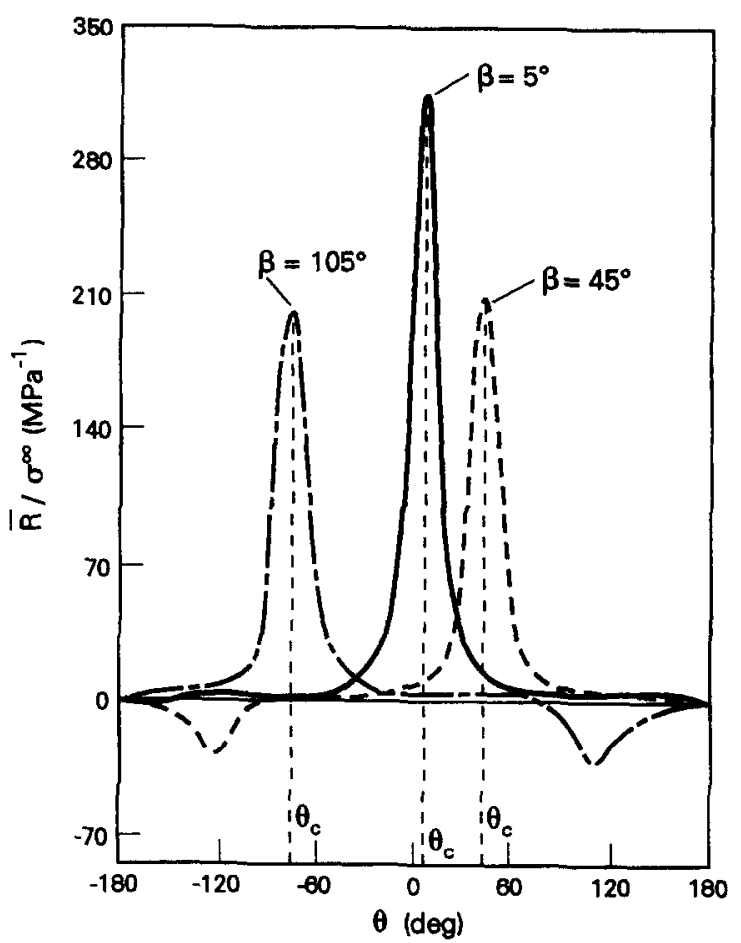

Fig. 4. Variation of $R$ as a function of $\theta$, for $\beta=5^{\circ}, 45^{\circ}$ and $105^{\circ}$.

$S=0.0998 \mathrm{GPa}$, which are the mechanical properties of unidirectional graphite fibre reinforced epoxy AS4/3501-6 and typifies the strongest anisotropy of stiffness and strength properties. For the sake of comparison, the curve of strain energy density, SED vs $\theta$ and the curve of normal stress ratio, $\bar{R}$ vs $\theta$ are also presented in Figs 3 and 4 respectively. From Fig. 2, it can be seen that the cracking angle $\theta_{c}$ predicted by SEDR criterion is not equal to the fibre direction $\beta$, but with a little deviation from it. In contrast with SEDR criterion, Fig. 4 indicates that the normal stress ratio criterion predicted the cracking direction $\theta_{c}$ being approximately equal to $\beta$. Unfortunately, the SED $-\theta$ curves predicted unsatisfactory results. To illustrate the dependency of $\theta_{c}$ on $\beta$, we plotted the curves of $\theta_{c}$ against $\beta$ predicted by SEDR and NSR criteria in Fig. 5 . It is clear that

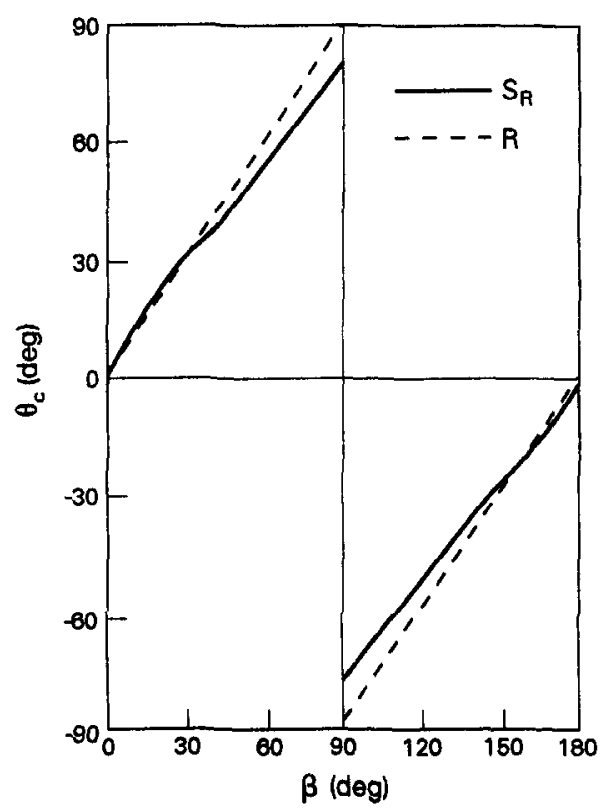

Fig. 5. Curves of cracking direction $\theta_{c}$ vs $\beta$, predicted by two criteria. 


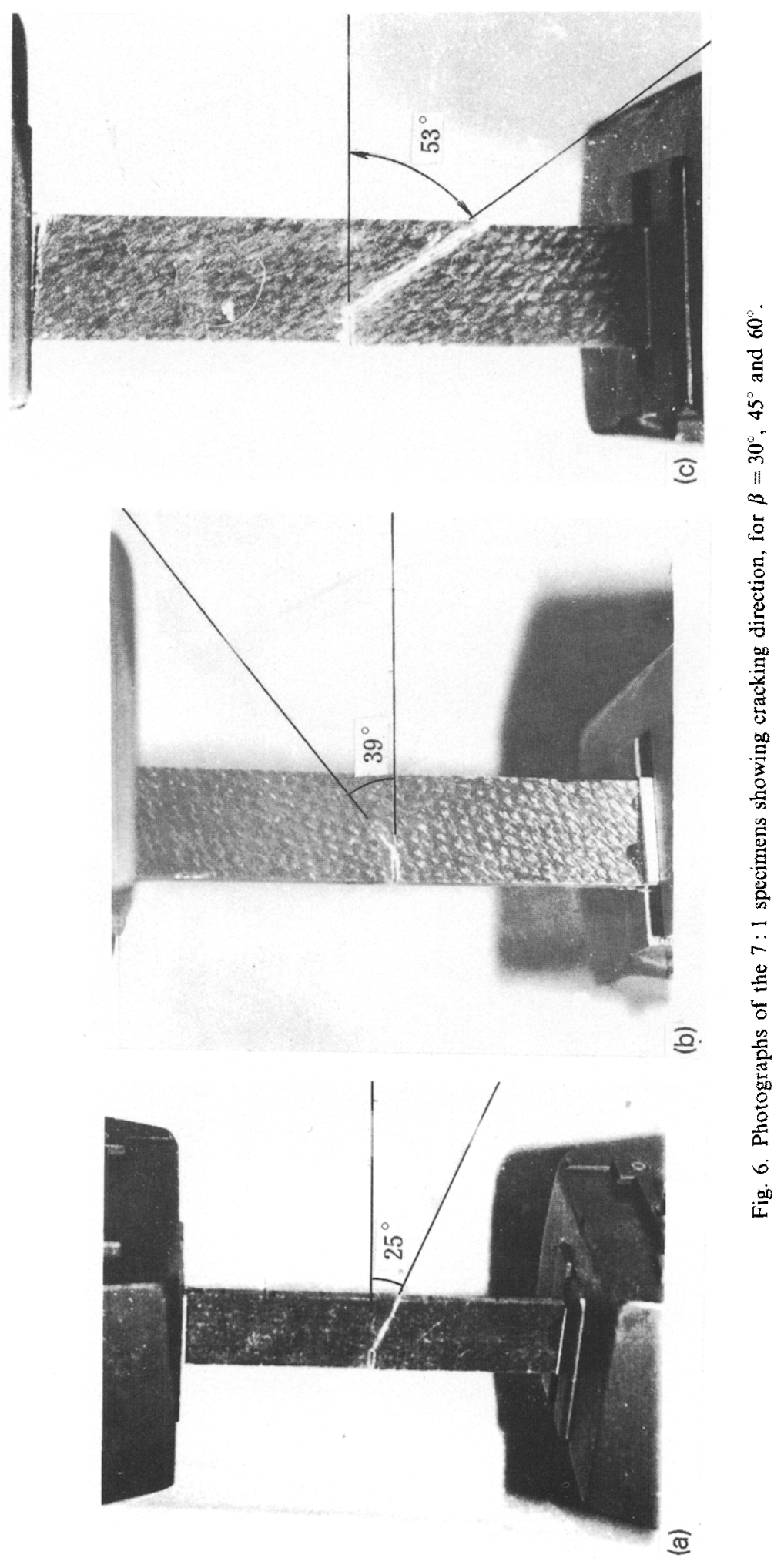




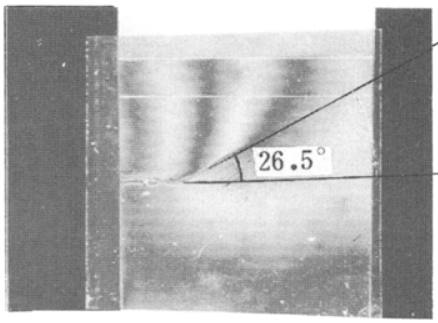

$\beta=30$

$\theta_{c}=26.5$

$7(\mathrm{a})$

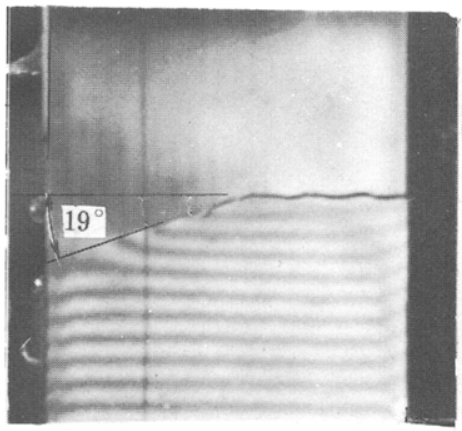

$\beta=30$

$\theta_{c}=19$

8(a)

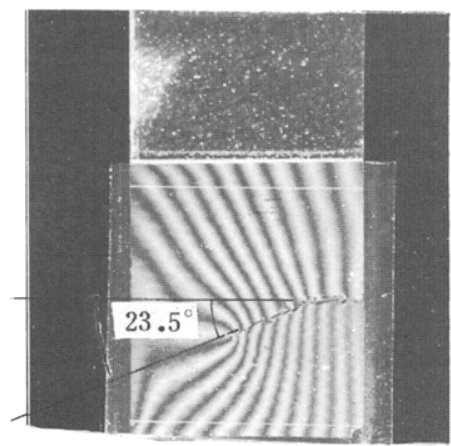

$\beta=30$

$\theta_{c}=23.5$

9(a)
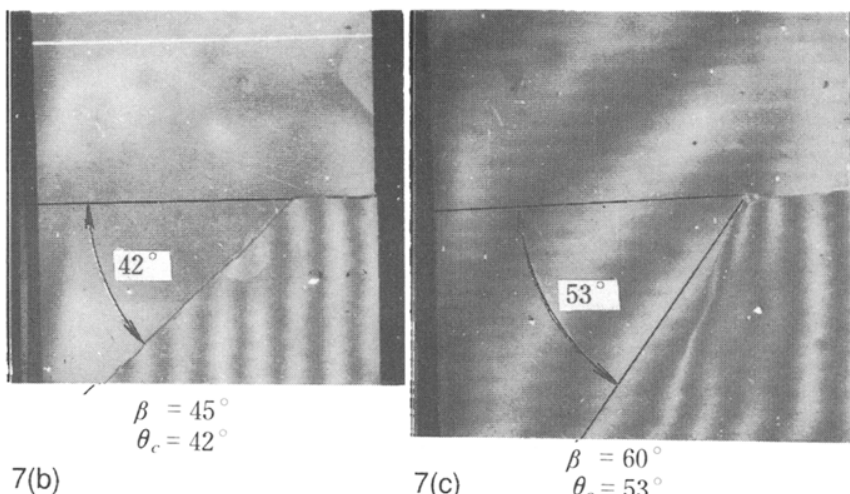

7(b)

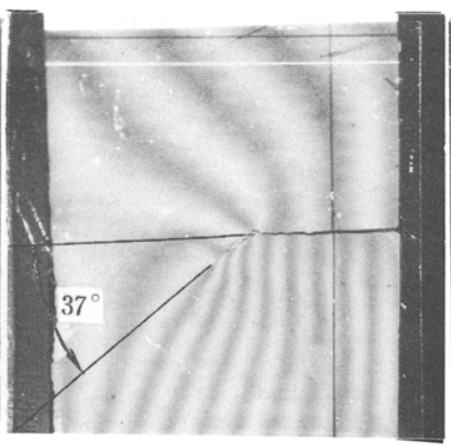

$\beta=45^{\circ}$
$\theta=37$

8(b)

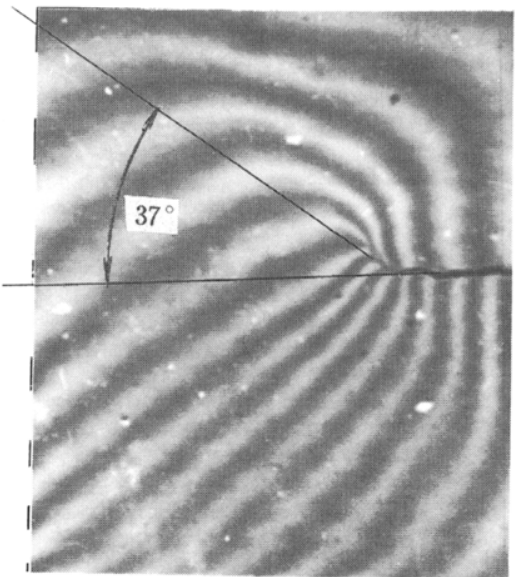

8(c)

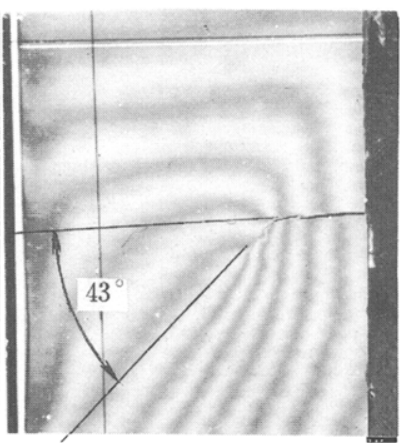

$\beta=60$

$\theta_{c}=43$

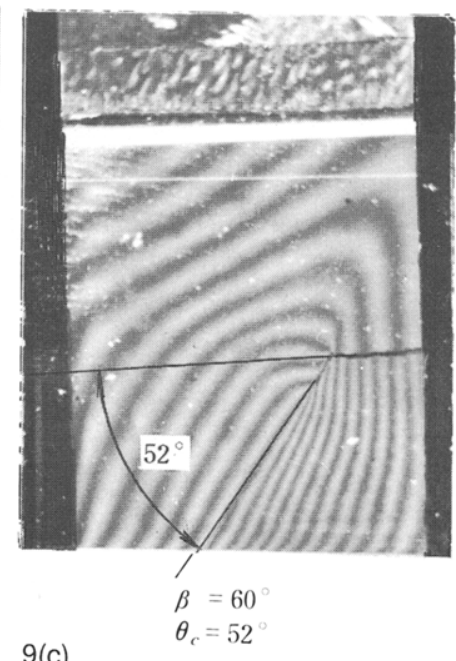

9(c)

$$
\begin{aligned}
& \beta=45^{\circ} \\
& \theta_{c}=37^{\circ}
\end{aligned}
$$$$
\theta_{c}=37
$$

Fig. 7. Moire striation photographs of the unidirectional specimens showing cracking direction, for $\beta=30^{\circ}, 45^{\circ}$ and $60^{\circ}$.

Fig. 8. Moire striation photographs of the $7: 1$ specimens showing cracking direction, for $\beta=30^{\circ}, 45^{\circ}$ and $60^{\circ}$.

Fig. 9. Moire striation photographs of the $4: 1$ specimens showing cracking direction, for $\beta=30^{\circ}, 45^{\circ}$ and $60^{\circ}$. 
Table 1. Material constants of the materials studied

\begin{tabular}{lccccccc}
\hline Reinforcement & $E_{11}(\mathrm{GPa})$ & $E_{22}(\mathrm{GPa})$ & $G_{12}(\mathrm{GPa})$ & $v_{12}$ & $X(\mathrm{MPa})$ & $Y(\mathrm{Mpa})$ & $S(\mathrm{MPa})$ \\
\hline Unidirectional & 26.11 & 8.63 & 2.76 & 0.345 & 663.6 & 10.1 & 22.8 \\
$7: 1$ fabric & 23.82 & 9.41 & 2.43 & 0.304 & $254.5+$ & 35.2 & 39.2 \\
$4: 1$ fabric & 20.23 & 8.67 & 2.45 & 0.260 & 401.8 & 64.3 & 48.8 \\
\hline
\end{tabular}

$+\mathrm{NB}$ : This value is lower than the expected, but several repeated tests yielded similar values.

Table 2. Comparison of predicted $\theta_{c}$ with experimental $\theta_{c}$ for unidirectional specimens $\left(\theta_{c}\right.$ in degrees)

\begin{tabular}{|c|c|c|c|c|c|}
\hline \multicolumn{2}{|l|}{ Specimen } & \multicolumn{3}{|c|}{ Predicted } & \multirow{2}{*}{$\frac{\text { Experimental }}{\text { Moire method }}$} \\
\hline Lay-up angle & $\beta$ & SEDR & NSR & Extended Tsai-Hill & \\
\hline $\begin{array}{l}60 \\
45 \\
30\end{array}$ & $\begin{array}{l}30 \\
45 \\
60\end{array}$ & $\begin{array}{l}27 \\
39 \\
52\end{array}$ & $\begin{array}{l}30 \\
45 \\
60\end{array}$ & $\begin{array}{l}26 \\
39 \\
52\end{array}$ & $\begin{array}{l}26.5 \\
42 \\
53\end{array}$ \\
\hline
\end{tabular}

when $\beta$ is small, the difference between predictions by the two criteria is quite small, whereas the deviation between them becomes larger with $\beta$.

\subsection{Experimental verifications}

The fracture experiments were carried out on the three kinds of glass fibre reinforced polyester resin (GRP), their reinforcements were: (a) unidirectional fibres; (b) glass fibre fabric with the ratio of warp fibre content to weft fibre content being $7: 1$ and (c) the glass fabric of warp/weft being 4:1. Before the fracture tests, some preliminary tests were done to measure the mechanical properties needed in the computations. The experimental results are listed in Table 1. It is obvious that the unidirectional GRP possesses the strongest anisotropy, the $4: 1$ fabric GRP possesses the weakest anisotropy and the anisotropy of $7: 1$ fabric GRP is intermediate.

The fracture tests were conducted by using the single edge notched specimens. The Instron1195 testing machine was used and the speed of the cross-head of the machine was $0.1 \mathrm{~mm} / \mathrm{min}$. For every kind GRP, three categories of specimens were manufactured and tested, i.e. their angles of the first material principal direction to the notch direction are $30^{\circ}, 45^{\circ}$ and $60^{\circ}$ respectively. In most cases, the cracking is a slow and gradual process. The cracking directions of all specimens were recorded with Moire interference technique, and the crack propagation direction of $7: 1$ specimens were also recorded by taking photographs.

The three photographs in Fig. 6 show the crack directions of $7: 1$ specimens with the three lay-up angles, i.e. $60^{\circ}, 45^{\circ}$ and $30^{\circ}$, which correspond to the three fibre directions: $\beta=30^{\circ}, 45^{\circ}$ and $60^{\circ}$, respectively. Figures 7-9 show the Moire striation photographs. They are for the unidirectional, $7: 1$ and $4: 1$ specimens respectively and have three lay-up angles each. It is clear that the Moire interference striations are broken by the crack. Comparing with ordinary photographing method, the Moire method is more sensitive and enables us record the crack initiation direction.

The cracking directions of all the 9 kinds of specimens were calculated with three criteria: namely, SEDR, NSR[2] and extended Tsai-Hill criteria[6]. The predicted results are compared with experimental ones as shown in Tables $2-4$. It can be seen that the predicted results are in reasonably

Table 3. Comparison of predicted $\theta_{c}$ with experimental $\theta_{c}$ for the $7: 1$ specimens $\left(\theta_{c}\right.$ in degrees)

\begin{tabular}{|c|c|c|c|c|c|c|}
\hline \multicolumn{2}{|l|}{ Specimen } & \multicolumn{3}{|c|}{ Predicted } & \multicolumn{2}{|c|}{ Experimental } \\
\hline Lay-up angle & $\beta$ & SEDR & NSR & Extended Tsai-Hill & Moire & Photography \\
\hline $\begin{array}{l}60 \\
45 \\
30\end{array}$ & $\begin{array}{l}30 \\
45 \\
60\end{array}$ & $\begin{array}{l}25 \\
36 \\
48\end{array}$ & $\begin{array}{l}29 \\
43 \\
57\end{array}$ & $\begin{array}{l}25 \\
37 \\
48\end{array}$ & $\begin{array}{l}19 \\
37 \\
43\end{array}$ & $\begin{array}{l}25 \\
39 \\
53\end{array}$ \\
\hline
\end{tabular}

Table 4. Comparison of predicted $\theta_{c}$ with experimental $\theta_{c}$ for the $4: 1$ specimens $\left(\theta_{c}\right.$ in degrees)

\begin{tabular}{|c|c|c|c|c|c|}
\hline \multicolumn{2}{|l|}{ Specimen } & \multicolumn{3}{|c|}{ Predicted } & \multirow{2}{*}{$\begin{array}{l}\text { Experimental } \\
\text { Moire method }\end{array}$} \\
\hline Lay-up angle & $\beta$ & SEDR & NSR & Extended Tsai-Hill & \\
\hline $\begin{array}{l}60 \\
45 \\
30\end{array}$ & $\begin{array}{l}30 \\
45 \\
60\end{array}$ & $\begin{array}{l}23 \\
34 \\
45\end{array}$ & $\begin{array}{l}29 \\
43 \\
50\end{array}$ & $\begin{array}{l}22.5 \\
33.5 \\
40\end{array}$ & $\begin{array}{l}23.5 \\
37 \\
52\end{array}$ \\
\hline
\end{tabular}




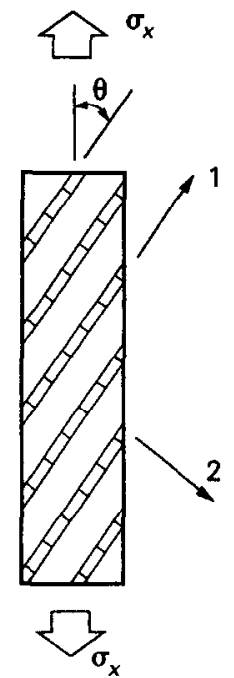

Fig. 10. Off-axial tension test scheme.

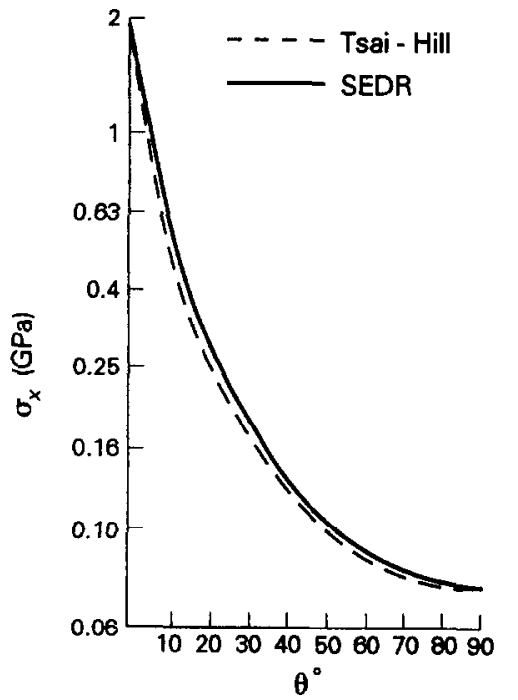

Fig. 11. Predicted curves of $\sigma_{x}$ vs $\theta$ by two criteria.

good agreement with measured ones. From Table 3, it can be noted that the cracking angle $\theta_{c}$ measured by Moire method is smaller than that by optical photographs. The reason seems to be that the optical photograph records the direction of well-developed crack, whereas Moire fringe gives the cracking direction in the early stage of fracture.

The comparison of Tables 2, 3 and 4 illustrates that for unidirectional GRP, the deviation of the cracking direction $\theta_{c}$ from the fibre direction is the smallest, the deviation of $\theta_{c}$ from $\beta$ for $4: 1$ specimens is the largest, with that of $7: 1$ specimens in the middle. This indicates the materials with stronger anisotropy of mechanical properties tends to crack along an angle closer to the fibre directions.

Comparing the three criteria used, it can be noted that the NSR criterion predicts the cracking direction $\theta_{c}$ nearly identical to the fibre direction $\beta$. The ignorance of shear strength effect in the NSR expression seems to be the explanation. The extended Tsai-Hill criterion yields $\theta_{c}$ similar to that of SEDR criterion proposed in the present paper. For all the three kinds of GRP, there is a common tendency, that is, when $\beta$ is small the deviation of $\theta_{c}$ from $\beta$ is small, and as $\beta$ rises the deviation becomes larger. This is in coincidence with the diagram shown in Fig. 5.

\subsection{Prediction of failure strength with $S E D R$ criterion}

In this section of the paper, the SEDR criterion used as failure criterion will be described. The off-axial tensile strength of the unidirectional fibre composite was analysed (see Fig. 10). According to eq. (8), the failure condition stipulated by the SEDR criterion can be written as:

$$
\frac{\cos ^{4} \theta}{X^{2}}+\frac{\sin ^{4} \theta}{Y^{2}}-\frac{v_{12}}{E_{11}} \sin ^{2} \theta \cos ^{2} \theta\left(\frac{E_{11}}{X^{2}}+\frac{E_{22}}{Y^{2}}\right)+\frac{\sin ^{2} \theta \cos ^{2} \theta}{S^{2}}=\frac{1}{\sigma_{x}^{2}} \text {. }
$$

For the sake of comparison, the failure condition deduced from Tsai-Hill criterion is also given as:

$$
\frac{\cos ^{4} \theta}{X^{2}}+\left(\frac{1}{S^{2}}-\frac{1}{X^{2}}\right) \cos ^{2} \theta \sin ^{2} \theta+\frac{\sin ^{4} \theta}{Y^{2}}=\frac{1}{\sigma_{x}^{2}}
$$

The material analysed is again the unidirectional graphite fibre reinforced epoxy $\mathrm{AS}_{4} / 3501-6$, whose constants have been given in the last section. The results are shown in Fig. 11 and Table 5, they

Table 5. Off-axial tensile strengths predicted by SEDR and Tsai-Hill criteria

\begin{tabular}{lcccccccccc}
\hline \multicolumn{1}{c}{ Fibre angle } & $0^{\circ}$ & $10^{\circ}$ & $20^{\circ}$ & $30^{\circ}$ & $40^{\circ}$ & $50^{\circ}$ & $60^{\circ}$ & $70^{\circ}$ & $80^{\circ}$ & $90^{\circ}$ \\
\hline Tsai-Hill criterion & 1983 & 546 & 274 & 178 & 130 & 103 & 86.8 & 77.1 & 71.9 & 70.3 \\
SEDR criterion & 1983 & 558 & 279 & 178 & 131 & 104 & 87.1 & 77.2 & 72.0 & 70.3 \\
\hline
\end{tabular}

NB: The unit of strength in this Table is $\mathrm{MPa}$. 
indicate that the failure strength predicted by SEDR criterion is intimately close to that by the Tsai-Hill criterion. Therefore, we may conclude that predicting failure strength with SEDR criterion is also successful.

\section{CONCLUDING REMARKS}

The validity of the SEDR criterion was verified with the fracture experiments of three kinds of GRP, which possess different degree of anisotropy of mechanical behaviour. The Moire interference fringe method and photograph observation were used to record the cracking direction. Through the comparison of predicted with experimental results, the SEDR criterion proposed by the present paper prove to be valid for predicting cracking direction in fibre composites. The calculations with NSR and the extended Tsai-Hill criteria were also conducted and yielded acceptable results. The cracking direction $\theta_{c}$ predicted by NSR criterion is closer to the fibre direction.

The theoretical comparison between the SEDR criterion and the well-known Tsai-Hill criterion indicates that the SEDR criterion can also be used as failure criterion for predicting failure strength of specimens without any stress saiser and discontinuities.

The SEDR criterion might be used to predict failure strength and crack growth direction in any kind of orthotropic materials.

Acknowledgements - This paper has been supported by the National Science Foundation and the Chinese Academy of Sciences under a special grant.

\section{REFERENCES}

[1] G. C. Sih and D. Y. Tzou, Discussion on "Criteria for brittle fracture in bi-axial tension" by S. K. Maiti and R. A. Smith. Engng Fracture Mech. 21, 977-982 (1983).

[2] M. A. Gregory and C. T. Herakovich, Predicting crack growth direction in unidirectional composites. J. Compos. Mater. 20, 67-85 (1986).

[3] G. C. Sih, Fracture mechanics of composite materials. Proc. 1st U.S.A.-U.S.S.R. Symp. Fracture Composite Mater., Riga, U.S.S.R., pp. 111-130 (1978).

[4] S. Y. Zhang, A note on fracture toughness of unidirectional composites. Int. J. Fracture 35, R57-R60 (1987).

[5] L. W. Tsai and S. Y. Zhang, Prediction of mixed-mode cracking direction in random short fibre composite materials. Composite Sci. Tech. 31, 97-110 (1988).

[6] S. Y. Zhang and L. W. Tsai, Extending Tsai-Hill and Norris criteria to predict cracking direction in orthotropic materials. Int. J. Fracture 40, R101-R104 (1989). 